\title{
Prostaglandin E1 Inhibited Diabetes- Induced Phenotypic Switching of Vascular Smooth Muscle Cells Through Activating Autophagy
}

\author{
Xing-Rong An ${ }^{a} \quad$ Xin $\mathrm{Li}^{\mathrm{a}} \quad$ Wei Wei ${ }^{\mathrm{a}} \quad$ Xiao-Xue Li ${ }^{\mathrm{b}} \quad$ Ming Xu \\ aDepartment of Clinical Pharmacy, School of Preclinical Medicine and Clinical Pharmacy, China \\ Pharmaceutical University, Nanjing, 'Department of Pharmacology, Southeast University School of \\ Medicine, Nanjing, China
}

\section{Key Words}

Diabetes • Phenotype switching • Autophagy • AKT • MTOR • PGE1

\begin{abstract}
Background/Aims: The phenotype switching of vascular smooth muscle cells (VSMCs) was associated with the onset or progression of the atherogenic process in type 2 diabetes mellitus (T2DM). Alprostadil (Prostaglandin E1, PGE1) as a bioactive drug had a protective effect on vascular function. However, it is unknown whether PGE1 inhibited the phenotype switching in VSMCs via autophagy, which played a protective role in the vascular complications of diabetes. Methods: The phenotype switching was induced by high glucose (HG, 25mM) in VSMCs, the protein expression was measured by western blot analysis and immunofluorescent staining. In vivo study, vascular lesion and dysfunction were produced in the rats fed with high fat diet (HFD) combined with low dose streptozotocin (STZ) administration. Results: The decrease of $\alpha$-SMA and the increase of vimentin, collagen I and proliferating cell nuclear antigen (PCNA) were found in HG-treated VSMCs. Along with more abundance of p62, autophagy markers LC3B and Beclin-1 significantly decreased in VSMCs exposed to HG. Such abnormal changes were significantly reversed by PGE1, which mimicked the role of autophagy activator rapamycin and was dramatically counteracted by 3-methyladenine, an autophagy inhibitor. Furthermore, PGE1 suppressed the phosphorylation of AKT and mTOR, which negatively regulated autophagy level in VSMCs. In vivo study, PGE1 remarkably improved the endotheliumindependent contraction of thoracic aorta and restored the expression of $\alpha$-SMA, osteopontin, LC3B, phosphorylated mTOR in the artery media of T2DM rats. Conclusion: These results demonstrated that PGE1 maintained the phenotype of VSMCs via the AKT/mTOR-dependent autophagy, which prevented diabetes-induced vascular complications.
\end{abstract}

X.-R. An and X. Li contributed equally to this work.

\begin{tabular}{ll}
\hline Ming Xu, MD, PhD & Department of Clinical Pharmacy, School of Preclinical Medicine and Clinical Pharmacy \\
& China Pharmaceutical University, 24 Tong jia Lane, P.O. Box 076, Nanjing, 210009 (China) \\
& E-Mail mingxu@cpu.edu.cn
\end{tabular}




\section{Cellular Physiology Cell Physiol Biochem 2018;50:745-756 \begin{tabular}{l|l|l} 
and Biochemistry & $\begin{array}{l}\text { DOI: 10.1159/000494240 } \\
\text { Published on } 2018 \text { The Author(s). Published by S. Karger AG, Basel } \\
\text { www.karger.com/cpb }\end{array}$
\end{tabular} \\ An et al.: PGE1 Inhibited Vascular Phenotypic Switching}

\section{Introduction}

Diabetes mellitus (DM) is an independent risk factor for cardiovascular diseases. VSMCs undergo phenotype switching in T2DM patients or diabetic rats, which might be associated with the onset or progression of atherogenic process [1]. However, the underlying molecular mechanisms mediating phenotypic change of VSMCs remain not entirely understood.

Autophagy is increasingly recognized as a critical determinant of VSMC biology involved in the pathogenesis of T2DM as a novel therapeutic target [2, 3]. In VSMCs, autophagy has been recognized as an important mediator of survival to protect cells against potential damage [4]. Many researches provided the evidences that the phenotypic switching in VSMCs was closed with autophagy [5]. However, it is controversial relationship between the phenotypic switching and autophagy. Therefore, there are great potential clinical implications in understanding the mechanisms underlying phenotypic switching in VSMCs in diabetic vascular lesion by pharmacological interventions.

PGE1 is widely applied in various clinical settings due to its biological functions such as vasodilatation, the inhibition of aggregation of platelet. Many studies showed that PGE1 could improve microcirculation [6], prevent ischaemia-reperfusion injury [7] and suppress angiogenesis [8], which indicated that PGE1 has been gradually extended from the treatment of chronic arterial occlusive diseases to the improvement of cardiovascular disorders. PGE1 also had a significant role in glucose and lipid metabolism in patients with T2DM. Our recent study showed that PGE1 could protect renal function via autophagy in diabetic nephropathy [9]. However, it has not been reported whether PGE1 has the potential protection on the phenotype in VSMCs via autophagy pathway.

The present study hypothesized that the regulation of autophagy is an important mechanism leading to the preventive effects of PGE1 on phenotypic switching in VSMCs. Given that HG could induce phenotypic transition of VSMCs in vitro, $25 \mathrm{mM}$ glucose was used in the current study. In vivo study, vascular lesion and dysfunction were produced in T2DM rats fed with HFD combined with low dose STZ administration. Our results supported that the inhibition of phenotype switching by PGE1 via autophagy was closely associated with the improvement of vascular dysfunction in T2DM.

\section{Materials and Methods}

\section{Isolation and culture of rat primary VSMCs}

VSMCs were obtained from aortas of male Sprague-Dawley (SD) rats as previously described [10]. Briefly, the thoracic aortas were separated and transferred to a dish with DMEM (Gibco, lifetechnologies, China). After removal of the fat tissue, the aorta was longitudinally cut and its intima was gently scraped to get rid of endothelial cells. Then, the aortas were cut into approximate $1 \mathrm{~mm}^{2}$ segments incubated in a chamber. DMEM containing 20\% fetal bovine serum (FBS, Gibco, lifetechnologies, USA) and 1\% antibiotic (Hyclone) was gently added in the chamber. VSMCs began to grow out from the edge of the tissue blocks after 8 days and became relatively confluent after 16 days. The identification of VSMCs was based on the morphology and the immunofluorescent staining of $\alpha$-SMA antibody with the positive rate $98.6 \%$. VSMCs with the passage 3-8 in the exponential phase were used for the experiments.

Western blot analysis

Western blot analysis was performed as described previously [9]. Briefly, 30 $\mathrm{gg}$ of total proteins from VSMCs were separated by $10 \%$ or $15 \%$ sodium dodecyl sulfate-polyacrylamide gel electrophoresis (SDSPAGE) at $80 \mathrm{~V}$ for $30 \mathrm{~min}$ and then $120 \mathrm{~V}$ for $1 \mathrm{~h}$. The protein was transferred to polyvinylidene fluoride membranes (PDVF membrane, Bio-Rad) at 200mA for $1 \mathrm{~h}$. After washing, the membranes were probed with the primary antibodies of $\alpha$-SMA, vimentin, Collagen I, PCNA, LC3B, Beclin1, p62, mTOR, p-mTOR, AKT, p-AKT, GAPDH (1:1000, Abways, China) overnight at $4{ }^{\circ} \mathrm{C}$, followed by Goat Anti-Rabbit IgG (1:5000, Abways, China) for $1.5 \mathrm{~h}$. The blot was detected by chemiluminescent systems with LumiGlo and Peroxide (1:1, Tanon). Densitometric analysis of the images was performed with Image J software (NIH, Littleton, CO, USA). 


\section{Cellular Physiology Cell Physiol Biochem 2018;50:745-756 \begin{tabular}{ll|l} 
aOI: 10.1159/000494240 & $\begin{array}{l}\text { O 2018 The Author(s). Published by S. Karger AG, Basel } \\
\text { www.karger.com/cpb }\end{array}$
\end{tabular} \\ An et al.: PGE1 Inhibited Vascular Phenotypic Switching}

Immunofluorescent staining of $\alpha$-SMA

VSMCs were fixed with $4 \%$ paraformaldehyde (PFA) for $15 \mathrm{~min}$ and permeabilized with $0.1 \%$ Triton $\mathrm{X}-100 / \mathrm{PBS}$ for $10 \mathrm{~min}$. Cells were incubated with anti- $\alpha$-SMA (1:200, Abways, China) primary antibody at $4^{\circ} \mathrm{C}$ overnight. After washing with PBS three times, VSMCs were incubated with Goat Anti-Rabbit IgG $(\mathrm{H}+\mathrm{L})$ Alexa Fluor 594 (1:200, Abways, China) for 1 hour at $37^{\circ}$, and then mounted in vector shield mounting medium with DAPI (Vector Laboratories Inc.). Cell was visualized on an olympus laser scanning confocal microscope (Carl Zeiss, LSM700). The optical density was determined by ImageJ software (NIH, Littleton, CO, USA).

\section{Establishing of type 2 diabetic rat model}

Adult male SD rats, weighing 230-250g, were purchased from Qinglongshan Lab Animal LtdهNanjing, China. Animal handling and experimental procedures were approved by the ethic committee of China Pharmaceutical University, in accordance with the Guidelines of Animal Experiment set by the Bureau of Sciences and Techniques of Jiangsu Province, China [NO.SYXK2007-0025]. SD rats were developed as a rat model of T2DM according to our previous study [11]. Rats were fed with the normal chow or HFD (22 $\mathrm{g} / \mathrm{d})$ that were purchased from Qinglongshan Lab Animal Ltd. Rats were divided into three groups: control, untreated diabetes and diabetes treated with PGE1(Meilunbio, China). In the first 6 weeks, rats in each group were given HFD alone with the normal group receiving regular chow only. On the beginning of 7 th week, rats fed with HFD received a single time intraperitoneal injection of STZ $(40 \mathrm{mg} / \mathrm{kg}$, dissolved in $\mathrm{pH}$ 4.5 citrate buffer, Sigma). Control rats only received an equivalent volume of citrate buffer. After the injection of STZ, all groups maintained their original diets.

Rats were recognized as diabetic rats while the fasting blood glucose (FBG) level reached $16.7 \mathrm{mM}$ after 1 week of STZ injection. In weeks 9-12, the interventions with PGE1 $(20 \mu \mathrm{g} / \mathrm{kg} / \mathrm{d})$ were conducted, however, rats in the groups of normal and untreated diabetes were given an equal volume of saline solution containing $0.6 \%$ ethanol. A gain of body weight was monitored at an interval of 3 days.

\section{Immunohistochemistry}

The paraffin sections of the aortic rings were incubated with anti- $\alpha$-SMA, anti-osteopontin, anti-LC3B, anti-p-mTOR antibody (1:200, Abways, China) overnight at 4 ]. After washing three times with PBS for 5 $\mathrm{min}$, the sections were incubated with goat anti-rabbit IgG for $2 \mathrm{~h}$ at room temperature. Then the horseradish enzyme labeled streptomyces biotin solution was added for 20 minutes at 370. DAB chromogen kit (Beyotime Biotechnology co, LTD.) was used for staining. The sections were counterstained with hematoxylin before examination under a light microscope.

\section{Vascular tension recording of rat thoracic aorta}

The endothelium of the rat aortas with a width of approximately 2-3 mm was removed and the isometric tensions were recorded with a PowerLab system (420S) (Taimeng, China). After equilibration in modified Krebs-Ringer bicarbonate solution, the aortic rings were contracted with phenylephrine $\left(10^{-6} \mathrm{M}\right)$ to induce submaximal contraction. No response to acetylcholine $\left(10^{-5} \mathrm{M}\right)$ in the denuded preparation indicated the effective denuding of the endothelium from aortic rings. Then the concentration-response curves for phenylephrine $\left(10^{-8}-10^{-5} \mathrm{M}\right)$ were obtained by the cumulative addition of phenylephrine in thoracic aorta, which was expressed as a percentage of the submaximal phenylephrine contraction.

\section{Statistical analysis}

Data are presented as the mean \pm SE. Significant differences between and within multiple groups were examined using analysis of variance for repeated measures, followed by Duncan's multiple-range test. Student's $t$ test was used to detect significant differences between two groups. $p<0.05$ was considered statistically significant. 


\section{Cellular Physiology Cell Physiol Biochem 2018;50:745-756

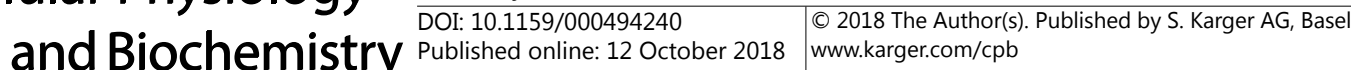 \\ An et al.: PGE1 Inhibited Vascular Phenotypic Switching}

\section{Results}

PGE1 reversed the phenotypic switching of VSMCs

Diabetes-induced vascular complication was associated with phenotypic modulation of VSMCs, switching from a contractile to a synthetic-proliferative phenotype [1]. In the preliminary study, we confirmed that $25 \mathrm{mM}$ glucose could induce the phenotypic switching of VSMCs and showed no significant effects in cell viability. To examine whether PGE1 inhibited phenotypic switching of VSMCs, quiescent cells were distributed into 4 groups: control and HG group with or without PGE1 $(0.05 \mathrm{ng} / \mathrm{ml})$ treatment for $24 \mathrm{~h}$. The protein levels of $\alpha$-SMA, vimentin, collagen I, and PCNA were tested by western blot analysis. As showed in Fig. 1A-D, HG significantly reduced the protein level of $\alpha$-SMA and enhanced the protein level of vimentin, PCNA and collagen I in VSMCs. Fluorescent analysis also showed that the fluorescent intensity of a contractile phenotype marker $\alpha$-SMA decreased in HGtreated VSMCs (Fig. 1E-1F). Further, ELISA assay (Caobenyuan Life Science, China) confirmed that HG promoted the secretion of collagen I in the cell supernatant (Fig. 1G). Such changes were effectively reversed by the treatment with PGE1, suggesting that PGE1 maintained VSMC phenotype under HG.

\section{PGE1 activated autophagy in VSMCs}

Autophagy is a survival mechanism to hyperglycemic toxicity [12]. Therefore, the enhancing autophagy would protect against HG-induced VSMCs phenotypic switching. Microtubule-associated protein 1 light chain 3 beta (LC3B) is an autophagy-related marker protein, which is recruited to autophagosomal membranes during the formation of autophagosomes. It was found that the transition of LC3B I to LC3B II significantly decreased in VSMCs exposed to HG (Fig. 2A). Similarly, HG led to a diminishment of Beclin-1 protein (Fig. 2B), which was required for the initiation of the autophagasome formation. The abundance of p62, a selective substrate of the autophagy degrading pathway was more accumulated in VSMCs pretreated with HG (Fig. 2C), suggesting that less p62 protein was degraded due to lowered autophage by HG. Such changes above were significantly reversed by PGE1 treatment, suggesting that PGE1 could recover the autophagy level under HG.

\section{Autophagy mediated phenotypic switch of VSMCS}

To investigate the role of autophagy in phenotypic switch in VSMCs, autophagy activator rapamycin and autophagy inhibitor 3-methyladenine were applied to treat with VSMCs. Cells were exposed to 50nM rapamycin or $5 \mathrm{mM} 3$-methyladenine with or without PGE1. As shown in Fig. 3A-3F, the upregulation of autophagy with rapamycin attenuated the phenotypic switch induced by HG manifested as the increase of $\alpha$-SMA expression, accompanied with the decrease of vimentin, PCNA, and collagen I. However, compared with PGE1 alone treated group, PGE1 combined with rapamycin showed no additive effects on the level of phenotypic makers in VSMCs. Conversely, we found that 3-methyladenine dramatically counteracted the protective role of PGE1 in phenotypic switching of VSMCs under HG (Fig. 4A-4F). Together, these results above suggested that autophagy indeed mediated phenotypic switch regulated by PGE1.

\section{Autophagy regulation is dependent on the AKT /mTOR signaling pathway}

It was documented that mTOR, a serine/threonine kinase, plays a crucial role in regulating autophagy [5]. To gain further insights into the molecular mechanisms underlying autophagy activation by PGE1, the possible involvement of mTOR was examined. As shown in Fig. 5A, PGE1 significantly suppressed the phosphorylation of mTOR under high glucose, which was similar with the effect of rapamycin (Fig. 5B). AKT is the upstream factor of mTOR to together form an AKT/mTOR signaling pathway involved in metabolism regulation [13]. 


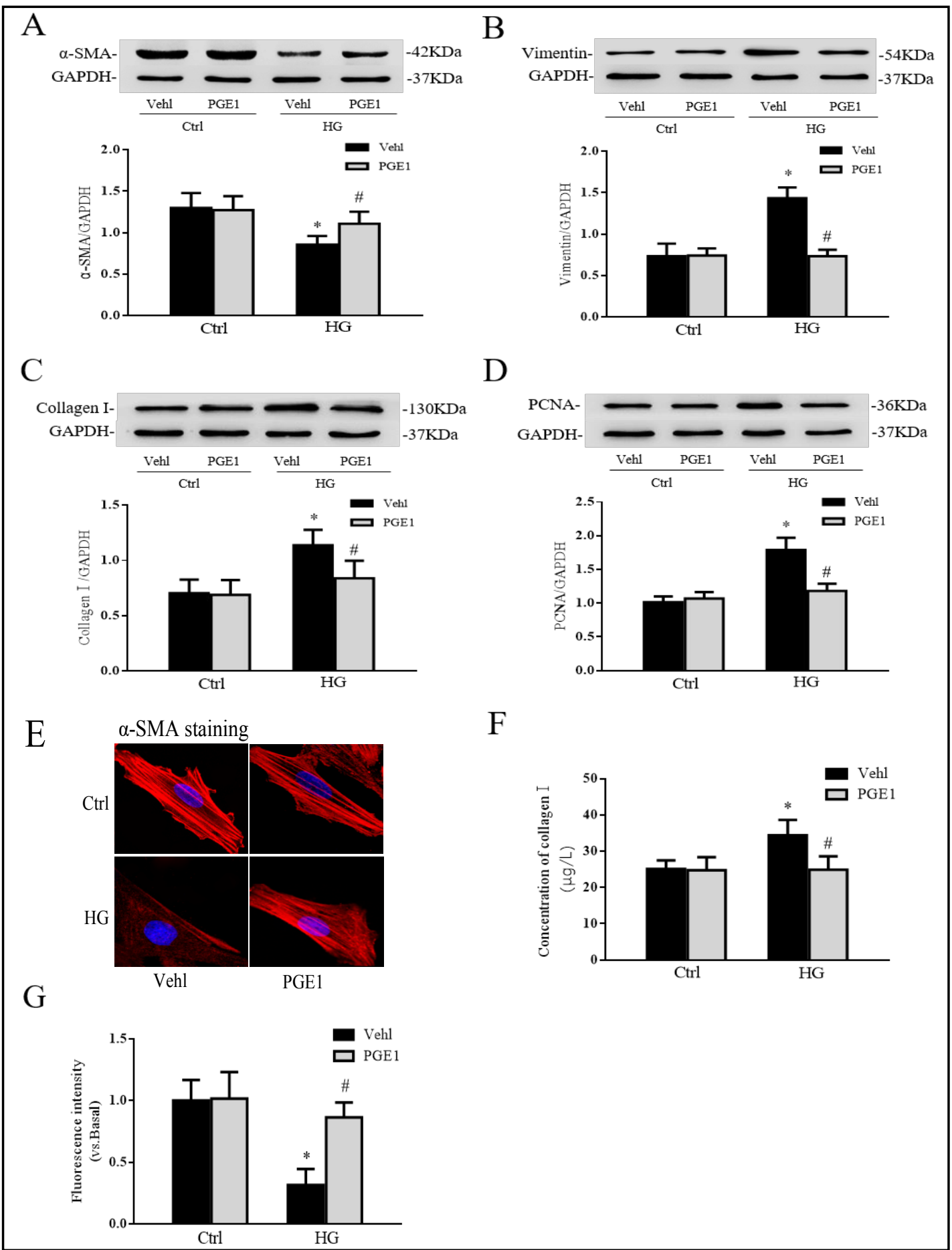

Fig. 1. PGE1 reversed the phenotypic switching of VSMCs VSMCs were incubated with high glucose (HG, $25 \mathrm{mM})$ for $48 \mathrm{~h}$, which were treated with PGE1 $(0.05 \mathrm{ng} / \mathrm{ml})$ for 24 hours. Representative Western blot gel documents and summarized data showed the protein expression of $\alpha$-SMA (A), vimentin (B), collagen I (C), and PCNA (D) in VSMCs. Typical fluorescent images (E) and summarized data (F) showed the mean fluorescent intensity of anti- $\alpha$-SMA followed by Alexa 594-labeled antibody. Summarized data showed the concentration of collagen I in the cell supernatant by ELISA assay (G). ${ }^{*} \mathrm{P}<0.05$ vs. Ctrl; ${ }^{\#} \mathrm{P}<0.05$ vs. HG treated group $(n=3)$. 
As shown in Fig. 5C, MK2206, a specific inhibitor of AKT phosphorylation led to the reduction of phosphorylation of mTOR in VSMCs. Further, PGE1 significantly inhibited the phosphorylation of AKT under HG (Fig. 5D). Therefore, the AKT/ mTOR signaling pathway was responsible for the regulation of PGE1 in autophagy.

PGE1 ameliorated arterial dysfunction in T2DM rats

FBG and fasting insulin (FINS) were assayed by glucose test kit (Jiancheng Bioengineering Institute, China) and rat insulin enzyme-linked immunosorbent assay kit (Halin Biological technology CO. LTD, China), respectively. The index of evaluating insulin sensitivity was Homeostatic Model Assessment (HOMA-IR), which was calculated as FBG (mmol/ $\mathrm{L}) \times$ FINS $(\mathrm{mU} / \mathrm{L}) / 22.5$. Following an injection of STZ in rats with HFD, FBG, FINS and HOMAIR were significantly elevated relative to control group, which were significant suppressed by PGE1 (20 $\mu \mathrm{g} / \mathrm{kg} / \mathrm{d}$ ) (Fig. 6A-6C).

Vascular response to phenylephrine was tested in thoracic aorta. Fig. 6D showed that the effects of PGE1 on contractile response to phenylephrine in aortic strips without endothelium from the HDF-fed rats following an injection of STZ. Compared to control rats, the contractile response to phenylephrine was significantly enhanced in the untreated diabetes mice. The impaired contraction was markedly improved in diabetic rats with PGE1 administration. These results implied that PGE1 may improve endothelium-independent contraction of thoracic aorta in diabetic rats.

The immunohistochemical analysis in Fig. $6 \mathrm{E}$ revealed that a significantly visible precipitation of $\alpha$-SMA was observed in the arterial media in the control group, but the definite decrease of these proteins could be noted in T2DM rats, which was significantly

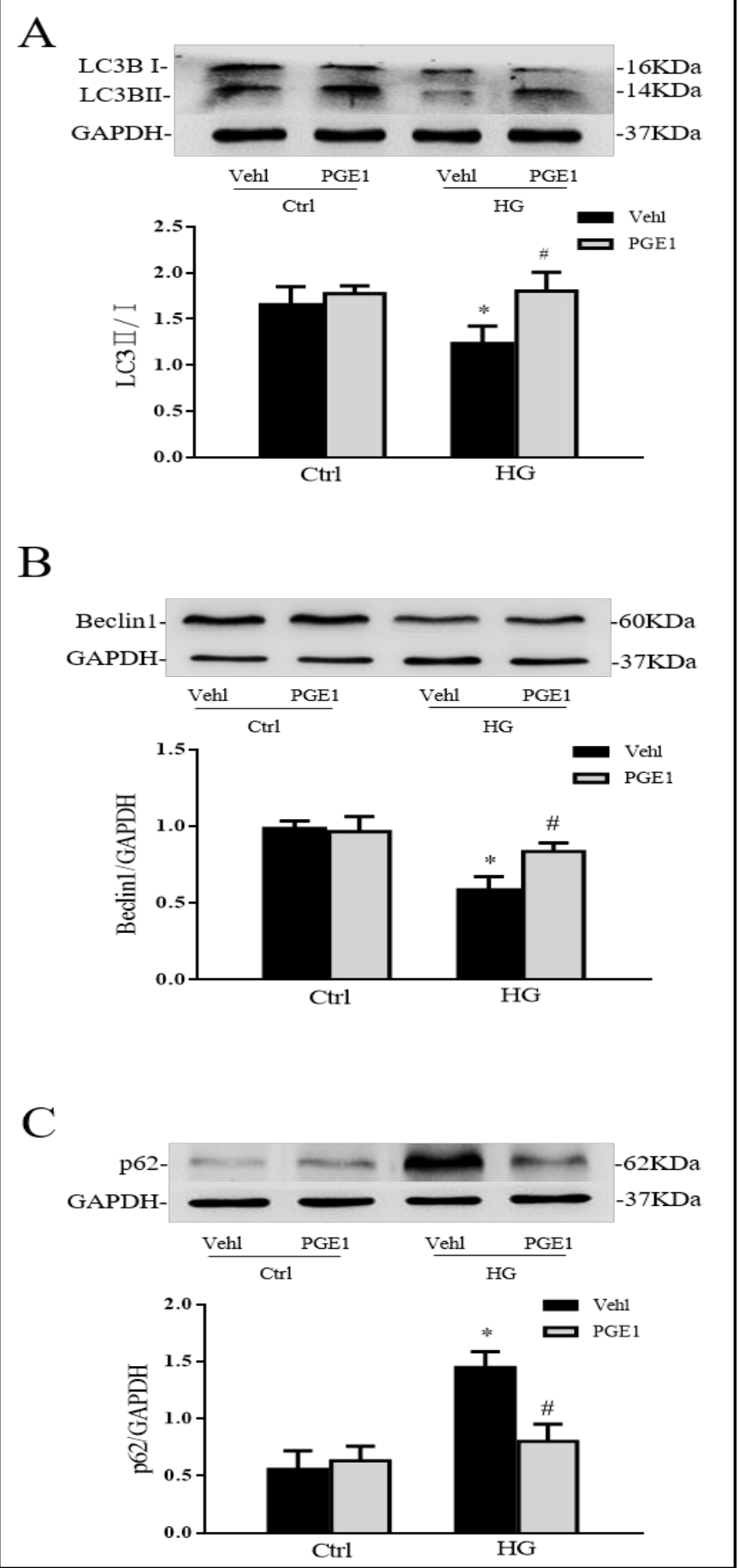

Fig. 2. PGE1 activated autophagy in VSMCs. Representative Western blot gel documents and summarized data showing the protein expressions of LC3B (A), Beclin-1 (B) and p62 (C) in VSMCs. ${ }^{*} \mathrm{P}<0.05$ vs. Ctrl; ${ }^{\#} \mathrm{P}<0.05$ vs. HG treated group $(\mathrm{n}=3)$ 


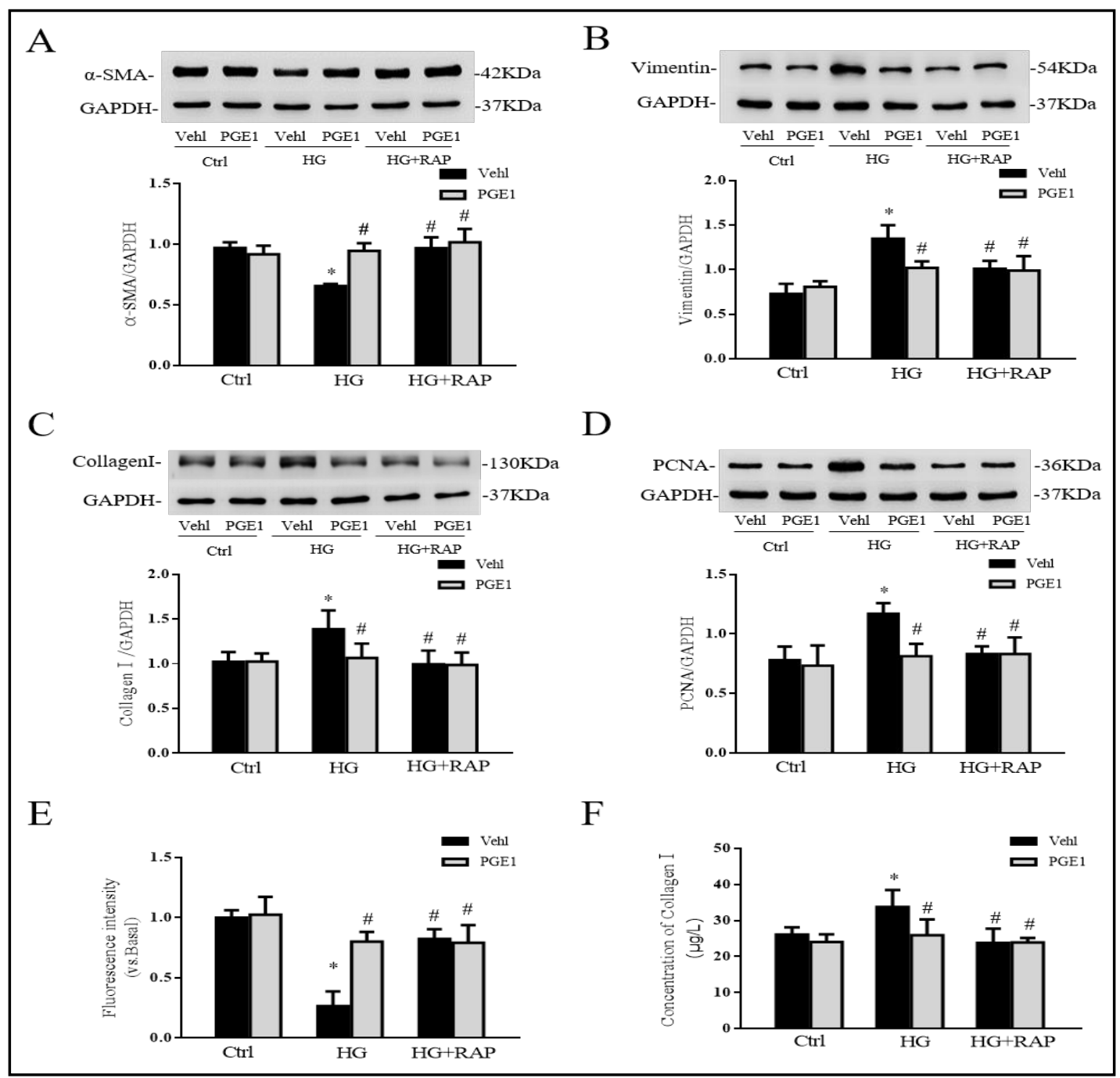

Fig. 3. Rapamycin mimicked the role of PGE1 on phenotypic switch. VSMCs were were exposed to rapamycin (RAP, 50nM) with or without PGE1 for 24 hours. Representative Western blot gel documents and summarized data showed the protein expression of $\alpha$-SMA (A), vimentin (B), collagen I (C), and PCNA (D) in VSMCs. Summarized data showed the mean fluorescent intensity of anti- $\alpha$-SMA followed by Alexa 594-labeled antibody (E). Summarized data showed the concentration of collagen I in the cell supernatant by ELISA assay (F). ${ }^{*} \mathrm{P}<0.05$ vs. Ctrl; ${ }^{\#} \mathrm{P}<0.05$ vs. HG treated group $(\mathrm{n}=3)$.

increased following PGE1 administration. On the contrary, PGE1 inhibited the osteopontin expression in arterial media compared with T2DM rats, which confirmed that PGE1 improved phenotypic switching in arterial VSMCs. In addition, the abnormal expression of LC3B and p-mTOR in the arterial media of T2DM rats were significantly restored by PGE1 administration. 


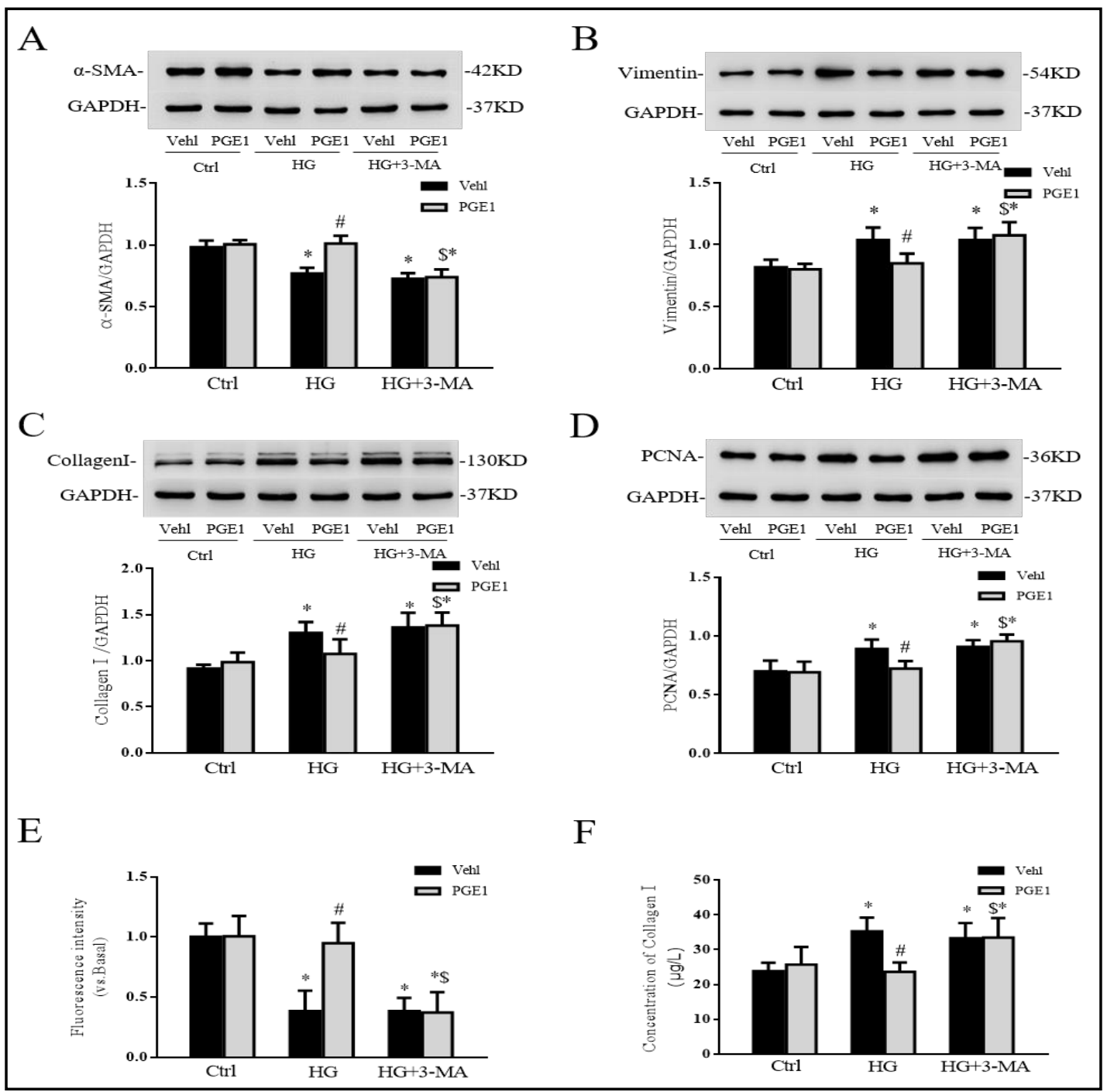

Fig. 4. 3-methyladenine counteracted the role of PGE1 on phenotypic switch. VSMCs were exposed to 3-methyladenine (3-MA, 5mM) with or without PGE1 for 24 hours. Representative Western blot gel documents and summarized data showed the protein expression of $\alpha$-SMA (A), vimentin (B), collagen I (C), and PCNA (D) in VSMCs. Summarized data showed the mean fluorescent intensity of anti- $\alpha$-SMA followed by Alexa 594-labeled antibody (E). Summarized data showed the concentration of collagen I in the cell supernatant by ELISA assay (F). ${ }^{*} \mathrm{P}<0.05$ vs. Ctrl; ${ }^{~} \mathrm{P}<0.05$ vs. HG treated group; ${ }^{\$} \mathrm{P}<0.05$ vs. HG+PGE1 treated group $(n=3)$. 


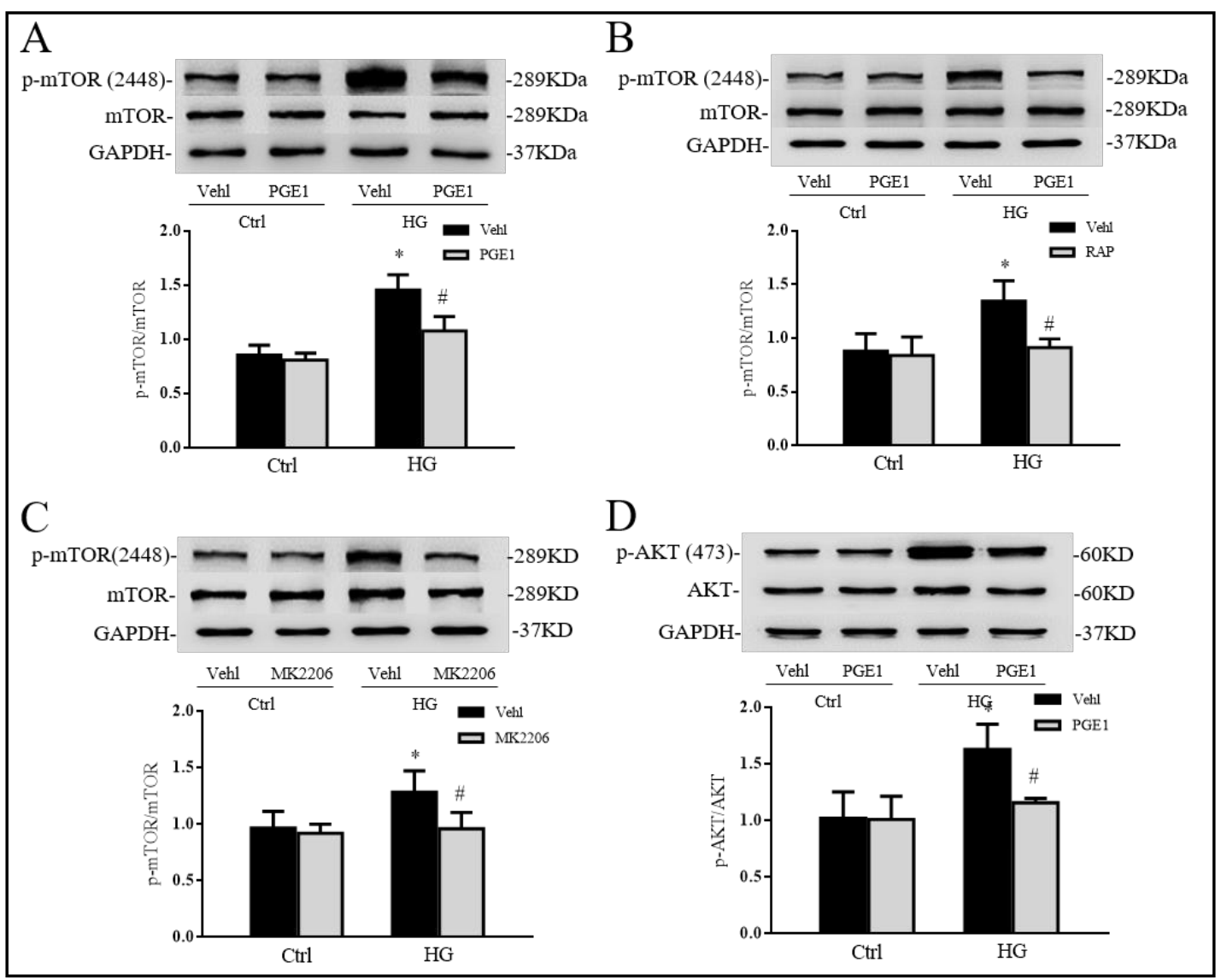

Fig. 5. PGE1 regulated autophagy via the AKT /mTOR signaling pathway. VSMCs were treated with PGE1, or rapamycin (RAP, 50nM), or MK2206 (5ng/ml) for 24 hours. Representative Western blot gel documents and summarized data showed the role of PGE1 (A) and rapamycin (B) and MK2206 (C) in the ratio of phosphorylated mTOR with mTOR. Representative Western blot gel documents and summarized data showed the role of PGE1 in the ratio of phosphorylated AKT with AKT (D). ${ }^{*} \mathrm{P}<0.05$ vs. Ctrl; ${ }^{\# P}<0.05$ vs. HG treated group $(n=3)$. 
Fig. 6. PGE1 ameliorated arterial dysfunction in T2DM rats. Diabetic rats were intervened with PGE1 $(20 \mu \mathrm{g} / \mathrm{kg} / \mathrm{d})$ for 4 weeks. Summarized data showed FBG (A), FINS (B) and HOMAIR (C). Vascular response to phenylephrine $\left(10^{-8}-10^{-5} \mathrm{M}\right)$ was determined in thoracic aorta, respectively (D). Representative immunohistochemistry images of $\alpha$-SMA, osteopontin (OPN), LC3B and p-mTOR in the arterial media (E). ${ }^{*} \mathrm{P}<0.05$ vs. Ctrl; ${ }^{\#} \mathrm{P}<0.05$ vs. T2DM rats $(\mathrm{n}=6)$.

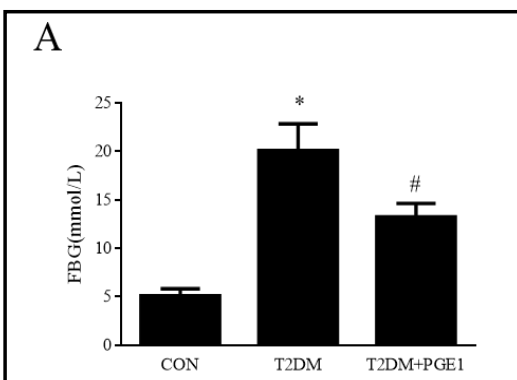

B
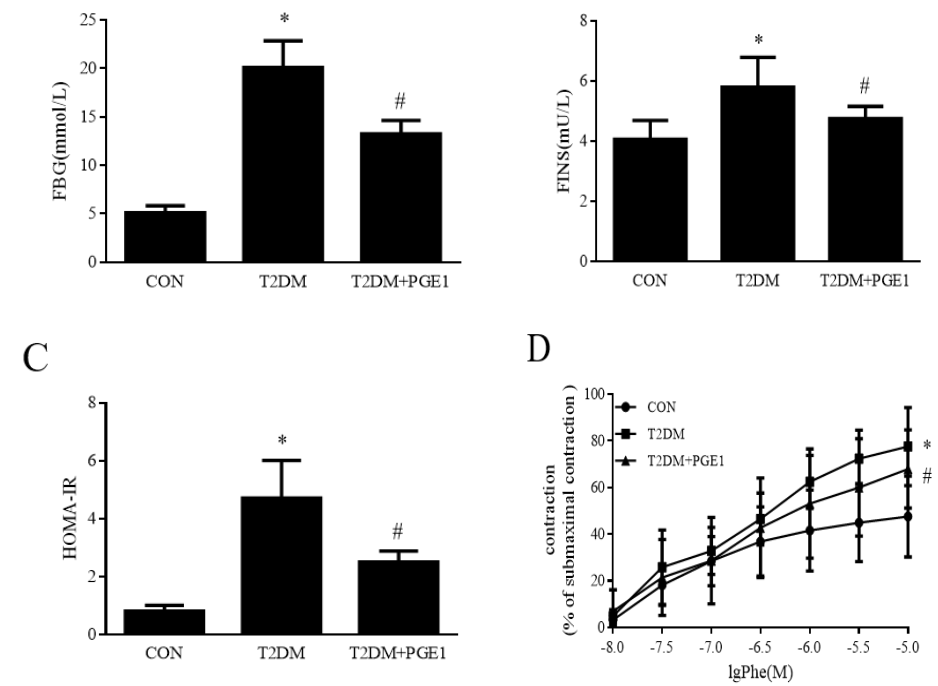

D

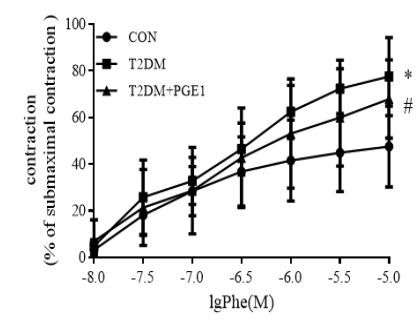

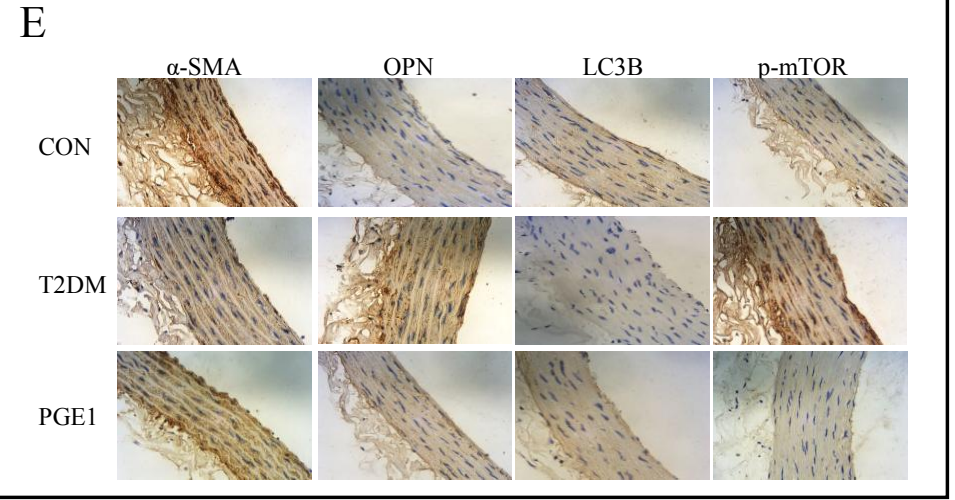

\section{Discussion}

The present study for the first time demonstrated that PGE1 reversed the phenotype change of VSMCs in T2DM rats. In response to various environmental stimuli, VSMCs convert from the differentiated phenotype to dedifferentiated phenotype characterized by the increased ability of proliferation/migration and the reduction of contractile ability, which is closely associated with some diseases, such as atherosclerosis and restenosis. It is also evident that VSMCs undertook phenotypic switching in T2DM [4], which was confirmed in the current study. Therefore, the reversion of the VSMC phenotypic switching is important to the prevention and therapy of diabetes-associated cardiovascular complications. For the first time, we demonstrated the protective role of PGE1 in restraining the phenotypic switching of VSMCs in vivo and vitro and the consequent improvement of vascular function in T2DM rats. It was demonstrated that PGE1 had the inhibiting effects on smooth muscle contraction [14]. Therefore, the present study suggested a novel pharmacological target of PGE1 on diabetic vascular complications.

Although the significant progress has been achieved over the past few years in our understanding of autophagy effects on vascular lesion $[15,16]$, it is still challenging to elucidate whether autophagy regulates phenotypic switching of VSMCs in T2DM. Our current results displayed that HG could inhibited the autophagy. Further, the upregulation of autophagy with rapamycin protected against phenotype change induced by HG. Conversely, 3-methyladenine exacerbated the phenotypic switching of VSMC under HG. Therefore, our 


\section{Cellular Physiology Cell Physiol Biochem 2018;50:745-756 \begin{tabular}{ll|l} 
DOI: 10.1159/000494240 & $\begin{array}{l}\text { O } 2018 \text { The Author(s). Published by S. Karger AG, Basel } \\
\text { www.karger.com/cpb }\end{array}$
\end{tabular} \\ An et al.: PGE1 Inhibited Vascular Phenotypic Switching}

results suggested that autophagy appeared to have a protective function in HG-induced vascular injury. It is controversial relationship between the phenotypic switching and autophagy in different pathological conditions. Rapamycin and its derivatives have been shown to prevent the phenotype switching and proliferation and promote the differentiation of VSMCs through a robust induction of autophagy. In contrast, autophagy induced by PDGF is essential for attaining the synthetic phenotype under oxidative stress in vascular lesions. Combined with our results, it is assumed that autophagy may have different roles in the phenotype transitions of VSMCs, depending upon the type of stimulators, the status of autophagy and the stages of diseases. The further study will be needed to investigate how autophagy regulated the phenotype of VSMCs in diabetes.

Here, we found that PGE1 mimicked the role of rapamycin on the phenotypic switching and was dramatically counteracted by 3-methyladenine. Our previous study demonstrated that inhibiting autophagy was responsible for the enhancement of FGF21 by PGE1 in palmitic acid-treated HK-2 cells [9]. The current study further demonstrated that autophagy was a new pharmacological target. A nutrient-sensing kinase mTOR overexpression significantly suppressed autophagic flux, which was involved in phenotypic switching of VSMCs. Consistently, our data indicated that HG significantly induced mTOR activation through the phosphorylation, which was inhibited by rapamycin. AKT is the upstream factor of mTOR [4], which was also demonstrated that mTOR inhibition by MK2206. Here, our results suggested that the AKT/mTOR signaling pathway was involved in the autophagy activation by PGE1 treatment under HG. It was reported that PGE2 induced the activation of the PI3K/AKT/ mTOR pathway in prostate cancer cells or mast cell chemotaxis [17]. Based on our results, it was suggested that AKT/mTOR signal pathway was responsible for the regulation of PGE1 on autophagy.

T2DM rats produced by HFD/low STZ provided a well-characterized model of vascular lesion and dysfunction. Here, we surprisingly found that PGE1 inhibited systemic insulin resistance and decreased the blood glucose in T2DM rats. The results from immunohistochemistry suggested that the phenotypic switching was formed and activated in the vessel media layer during T2DM accompanied with autophagy inhibition. PGE1 medication indeed ameliorated the phenotypic transformation of VSMCs, which was associated the activation of the mTOR-dependent autophagy pathway during T2DM. Further, the endothelium-independent contraction of aortic rings responding to phenylephrine was significantly impaired in isolated aorta in T2DM rats, whereas the impairment could be prevented by PGE1 medication. Accordingly, the autophagy mainly showed a protective role in diabetes-induced vascular dysfunction. It was consistent with the report that rapamycin protected against left ventricular dysfunction in diabetic rat [18].

\section{Conclusion}

Our current study provided the evidences that PGE1 could reverse the phenotypic switching of VSMCs and improved the vascular function by up-regulating autophagy in T2DM rats. Furthermore, the activation of autophagy was dependent on the AKT/mTOR pathway signaling. This study provided a novel pharmacological application of PGE1 and a new therapy strategy to the onset and development of diabetic macrovascular complications.

\section{Acknowledgements}

This work was supported by the National Natural Science Foundation of China (81570413 and 81773732) and Natural Science Foundation of Jiangsu Province (BK20161461). 


\section{Cellular Physiology Cell Physiol Biochem 2018;50:745-756 \begin{tabular}{ll|l} 
DOI: 10.1159/000494240 & $\begin{array}{l}\text { O } 2018 \text { The Author(s). Published by S. Karger AG, Basel } \\
\text { www.karger.com/cpb }\end{array}$
\end{tabular} \\ An et al.: PGE1 Inhibited Vascular Phenotypic Switching}

\section{Disclosure Statement}

The authors declare to have no competing interests.

\section{References}

1 Zhang X, Li R, Qin X, Wang L, Xiao J, Song Y, Sheng X, Guo M, Ji X: Sp1 Plays an Important Role in Vascular Calcification Both In Vivo and In Vitro. J Am Heart Assoc 2018;7:pii:e007555.

2 Vindis C: Autophagy: an emerging therapeutic target in vascular diseases. Br J Pharmacol 2015;172:21672178. Choi AM, Ryter SW, Levine B: Autophagy in human health and disease. N Engl J Med 2013;368:651-662. Nussenzweig SC, Verma S, Finkel T: The role of autophagy in vascular biology. Circ Res 2015;116:480-488. Wei YM, Li X, Xu M, Abais JM, Chen Y, Riebling CR, Boini KM, Li PL, Zhang Y: Enhancement of autophagy by simvastatin through inhibition of Rac1-mTOR signaling pathway in coronary arterial myocytes. Cell Physiol Biochem 2013;31:925-937.

6 Hong L, Zhang J, Shen J: Clinical efficacy of different doses of lipo-prostaglandin E1 in the treatment of painful diabetic peripheral neuropathy. J Diabetes Complications 2015;29:1283-1286.

7 San Norberto Garcia EM, Taylor JH, Cenizo N, Vaquero C: Beneficial effects of intra-arterial and intravenous prostaglandin E1 in intestinal ischaemia-reperfusion injury. Interact Cardiovasc Thorac Surg 2014;18:466474.

8 Cattaneo MG, Pola S, Deho V, Sanguini AM, Vicentini LM: Alprostadil suppresses angiogenesis in vitro and in vivo in the murine Matrigel plug assay. Br J Pharmacol 2003;138:377-385.

-9 Wei W, An XR, Jin SJ, Li XX, Xu M: Inhibition of insulin resistance by PGE1 via autophagy-dependent FGF21 pathway in diabetic nephropathy. Sci Rep 2018;8:9.

10 Chi J, Meng L, Pan S, Lin H, Zhai X, Liu L, Zhou C, Jiang C, Guo H: Primary Culture of Rat Aortic Vascular Smooth Muscle Cells: A New Method. Med Sci Monit 2017;23:4014-4020.

11 Li Q Su J, Jin SJ, Wei W, Cong XD, Li XX, Xu M: Argirein alleviates vascular endothelial insulin resistance through suppressing the activation of Nox4-dependent 02(-) production in diabetic rats. Free Radic Biol Med 2018;121:169-179.

12 Kobayashi S, Xu X, Chen K, Liang Q: Suppression of autophagy is protective in high glucose-induced cardiomyocyte injury. Autophagy 2012;8:577-592.

13 Perl A: Activation of mTOR (mechanistic target of rapamycin) in rheumatic diseases. Nat Rev Rheumatol 2016;12:169-182.

14 Michibayashi T: Inhibitory action of prostaglandin E1 on smooth muscle contraction and calcium responses. Prostaglandins 1978;15:803-812.

15 Li X, Xu M, Pitzer AL, Xia M, Boini KM, Li PL, Zhang Y: Control of autophagy maturation by acid sphingomyelinase in mouse coronary arterial smooth muscle cells: protective role in atherosclerosis. J Mol Med (Berl) 2014;92:473-485.

16 Xu M, Li XX, Chen Y, Pitzer AL, Zhang Y, Li PL: Enhancement of dynein-mediated autophagosome trafficking and autophagy maturation by ROS in mouse coronary arterial myocytes. J Cell Mol Med 2014;18:21652175.

17 Vo BT, Morton D, Jr., Komaragiri S, Millena AC, Leath C, Khan SA: TGF-beta effects on prostate cancer cell migration and invasion are mediated by PGE2 through activation of PI3K/AKT/mTOR pathway. Endocrinology 2013;154:1768-1779.

18 Zhou X, Li C, Lu X: Autophagy activation by rapamycin improves left ventricular function in diabetic rats. Int J Cardiol 2013;168:4429-4431. 